\title{
POSITION OF COMPACT HYPERSURFACES OF THE $n$-SPHERE
}

\author{
JAMES R. WASON ${ }^{1}$
}

\begin{abstract}
Let $S^{n}$ be the Euclidean sphere of dimension $n$. Let $p$ and $q$ be antipodal points on $S^{n}$, and, for nonnegative $h$, let $C(p, h), C(q, h)$ be the hyperspheres of constant mean curvature $h$ centered at $p$ and $q$, respectively. Then any closed hypersurface in $S^{n}$ with mean curvature bounded by $h$ must have a point in the 'tropical' region bounded by $C(p, h)$ and $C(q, h)$.
\end{abstract}

1. Introduction. Let $S^{n}$ be the $n$-sphere with the standard Riemannian metric induced by inclusion in $R^{n+1}$. For $p \in S^{n}$, let $C(p, k)$ be the $(n-1)$ sphere of constant mean curvature $k$, centered at $p$. Let $D(p, k)$ be the component of $S^{n}-C(p, k)$ containing $p$. We prove:

1.1 TheOREM. Let $M$ be a hypersurface in $S^{n}$ which is smooth, compact, and without boundary. Let $H$ be the mean curvature function on $M$. If $|H| \leqslant k$, then for any two antipodal points $p$ and $q$ in $S^{n}$, there is a point of $M$ lying in the set $A(p, q, k)=S^{n}-(D(p, k) \cup D(q, k))$.

Note that the boundary of $A(p, q, k)$ is just $C(p, k) \cup C(q, k)$. If $M$ is minimal, then $H \equiv 0$, and we have

1.2 COROLlarY. Let $M$ be a compact, oriented, minimal hypersurface without boundary in $S^{n}$. Then $M$ must intersect each great $(n-1)$-sphere.

The Corollary may also be proved using methods developed by $\mathrm{H}$. B. Lawson [2].

2. Proof of the Theorem. We prove first the following

LEMMA. If $|H| \leqslant k$, then there is a point of $M$ lying in $S^{n}-D(p, k)$.

Proof. Suppose $M$ lies entirely in $D(p, k)$. Since $M$ is compact, then it must also lie in the closure of $D(p, r)$ with $r>k$. Shrink $D(p, r)$ until $C(p, r)$ first touches $M$. Then at some point $m, M$ is tangent to $C(p, r)$. Since $M$ is tangent from within $D(p, r)$, it follows that $H(m) \geqslant r>k$. But this contradicts $|H| \leqslant k^{2}$

The Theorem now follows by application of the Lemma to the antipodal points $p$ and $q$.

Received by the editors February 20, 1976 and, in revised form, April 4, 1977.

AMS (MOS) subject classifications (1970). Primary 53C20.

'Work partially supported by NSF grant GP22928.

${ }^{2}$ The author would like to thank the referee for this abbreviation of his original argument. 


\section{REFERENCES}

1. S. Kobayashi and K. Nomizu, Foundations of differential geometry, Interscience, New York, 1963.

2. H. B. Lawson, Jr., The global behavior of minimal surfaces in $S^{n}$, Ann. of Math. (2) 92 (1970), 224-237.

Wellesley, Wellesley, MassachusetTs 02181 